\title{
WISC-R Verbal and Performance IQ Discrepancy in an Unselected Cohort: Clinical Significance and Longitudinal Stability
}

\author{
Terrie E. Moffitt \\ University of Wisconsin, Madison
}

\author{
P. A. Silva \\ Dunedin Multidisciplinary Health and \\ Development Research Unit \\ University of Otago, Dunedin, New Zealand
}

\begin{abstract}
This study examined children from an unselected birth cohort who had Wechsler Intelligence Scale for Children-Revised (WISC-R) verbal and performance IQ discrepancies that placed them beyond the 90 th percentile. It was hypothesized that, relative to their cohort peers, these children would be characterized by greater frequency of perinatal difficulties, early childhood neurological abnormalities, health problems of neurological significance, and concussion. Additionally, it was hypothesized that such children would exhibit more behavior problems, lower academic achievement, and poorer motor skills. Generally, the null hypotheses were not rejected by the results. Longitudinal investigation of the stability of the verbal and performance IQ discrepancy revealed that about $23 \%$ of discrepant cases were discrepant at two or more ages. Depressed verbal IQ relative to performance IQ was found to be more common than the reverse pattern. Children with performance IQ greater than verbal IQ scored significantly lower than children for whom this pattern was reversed on measures of academic achievement. Results show that cautious interpretation is needed of verbal and performance IQ discrepancy in the general (nonneurological) assessment setting.
\end{abstract}

Administration of the Wechsler Adult Intelligence Scale-Revised (WAIS-R; Wechsler, 1981) and the Wechsler Intelligence Scale Revised for Children (WISC-R; Wechsler, 1974) in the context of neuropsychological assessment has become widely accepted clinical practice. Perhaps one of the most frequently interpreted measures obtained from these tests is the discrepancy between verbal IQ (VIQ) and performance IQ (PIQ). Initial evidence for wide VIQ-PIQ discrepancy as an indicator of cognitive pathology was established by a series of articles by Reitan and Klove (Klove, 1959; Reitan, 1955; Klove \& Reitan, 1958) and was subsequently supported by numerous studies (see Matarazzo, 1972, Table 13.1).

The WISC VIQ-PIQ discrepancy has been associated specifically with a variety of verbal, motor, perinatal, and neurological problems in reports by Holroyd (1968), Black (1974), Rutter, Graham, and Yule (1970), and Dennis et al. (1981). These results have been reinforced by statements from popular texts. For example, Kolb and Whishaw wrote that "a difference of more than 10 points between the verbal and performance scores is usually taken to be a clinically significant difference, although statistically this interpretation is somewhat liberal" (1985, p. 674), and Lezak noted that "a pattern of clear-cut differences

The Dunedin Multidisciplinary Health and Development Research Unit is supported by the Medical Research Council of New Zealand and by the New Zealand Departments of Education and Health. The first author's work was supported by United States Public Health Service Grant 1 R23 MY-39994-0I from the Antisocial and Violent Behavior Branch of the National Institute of Mental Health.

Correspondence concerning this article should be addressed to Terrie E. Moffitt, Department of Psychology, University of Wisconsin, 1202 West Johnson Street, Madison, Wisconsin 53706. between subtests involving primarily verbal functions on the one hand and those involving primarily visuospatial functions on the other is likely, but not necessarily, a product of lateralized brain injury" (1983, p. 251).

Although Wechsler's intelligence scales were not originally designed as neuropsychological tests, the verbal and performance IQ scores fortuitously approximate measures of functions that are primarily lateralized in the two cerebral hemispheres (Kolb \& Whishaw, 1985). Because of this lateralized representation, the direction of the discrepancy may be useful for making diagnostic hypotheses about lesion loci. Guertin, Ladd, Frank, Rabin, and Hiester (1966) reviewed a number of studies suggesting that lower PIQ stores are indicative of righthemisphere pathology, whereas lower VIQ scores are indicative of damage to the left hemisphere, findings that are in agreement with the earlier reports of Reitan (1955) and of Klove and Rei$\tan$ (1958). More recently, Uzzell, Zimmerman, Dolinskas, and Obrist (1979) reported differential effects on VIQ and PIQ scores corresponding to lateralization of intracranial hematoma or hemorragic contusion.

Although Wechsler (1974) stated that a VIQ-PIQ score difference of 15 points is important and although Cronbach (1961) agreed that the VIQ-PIQ score has an estimated reliability $(.74$ at the $99 \%$ level of confidence) "high enough to justify drawing conclusions about the person whose verbal and performance IQs differ by 15 points or so" (p. 198), Kaufman (1980) argued compellingly for limiting diagnostic interpretation to wider discrepancies. He pointed out that one quarter of the WISC-R standardization sample had VIQ-PIQ discrepancy scores of 15 or more and cautioned the clinician to use a "statistical abnormality" criterion for diagnostic or educational purposes. He suggested a criterion of ViQ-PIQ $= \pm 19$ (i.e., less than $15 \%$ prevalence) or of VIQ-PIQ $= \pm 22$ (i.e., less than $10 \%$ prevalence). 
Despite the widespread clinical practice of VIQ-PIQ score interpretation and despite its seemingly substantial research base, the validity of VIQ-PIQ score use as an indicator of cognitive dysfunction is controversial. Numerous studies have found no relation between VIQ-PIQ scores and a variety of sources of brain dysfunction or neuropathological states (Bortner, Hertzig, \& Birch, 1972; Braun \& Brane, 1971; Caputo, Edmonton, L'Abate, \& Rondberg, 1963; Fedio \& Mirsky, 1969; Pihl, 1968; Wallace \& Cull, 1979). Other studies have found the direction of the VIQ-PIQ score discrepancy to have less lateralizing potential than previously thought (Dennis, 1985; Kane, Parsons, \& Goldstein, 1985; Matarazzo, 1972; Russell, 1979; Todd, Coolidge, \& Satz, 1977).

Rutter (1983) argued that "discrepancy indexes are of no value for the individual diagnosis of brain damage" (p. 190), basing his assertion primarily on the problem of differing base rates for brain damage and normality in the population. This assertion depends on the assumption that extreme VIQ-PIQ scores can be found in normal children. Are there significant numbers of normal children in the general population who show wide VIQ-PIQ score discrepancy? Or do most children who show large VIQ-PIQ score discrepancy have some (even if minor) history suggestive of possible central-nervous-system disorder?

Examination of the true rates of association of VIQ-PIQ scores with normal status and with compromised cognitive status due to acquired or congenital brain disorder requires study of an unselected sample of children. One such study (Bishop \& Butterworth, 1980) investigated the relation between VIQ-PIQ score at two ages ( $41 / 2$ years and $81 / 2$ years) and perinatal history, reading disability, and childhood neurological disease in 139 children from a general medical practice. The study found only weak associations between reading problems, perinatal problems, and VIQ-PIQ score at one of the two test ages. However, only 3 children had VIQ-PIQ scores over 24 points, and perinatal and neurological measures were retrospective.

The present research examines the clinical significance of VIQ-PIQ scores longitudinally in the context of a large unselected birth cohort using prospective measures of perinatal problems and neurological disorder along with measures of possible central nervous system insult, academic abilities, and behavior problems temporally concurrent with repeated WISC-R assessments. As stated by Bishop and Butterworth (1980), we are interested not in whether children with known brain damage have elevated rates of wide VIQ-PIQ score discrepancy but in whether a statistically abnormal VIQ-PIQ score signals clinically abnormal status for a child from the general population. This study attempted to determine whether there is any justification for interpreting a wide VIQ-PIQ score discrepancy as presumptive evidence of possible brain dysfunction as defined by nervous system insult history, early neurological abnormality, or perinatal problems. It also asked whether abnormal VIQ-PIQ score discrepancy portends adverse consequences for children's academic, behavior, and motor-skill status.

\section{Method}

\section{Subjects}

Subjects were children who were involved in the Dunedin (New Zealand) Multidisciplinary Health and Development Study. The cohort was fully described by McGee and Silva (1982). Briefly, the study longitudinally investigated the health, development, and behavior of a cohort of children born between April 1, 1972 and March 31. 1973 in Dunedin, New Zealand. Perinatal data were initially obtained, and when the children were traced at 3 years of age, 1,139 children were eligible for inclusion by their residence in the province of Otago. Of these. $1.037(91 \%)$ were assessed, and thereafter assessments occurred every 2 years, with 991 children assessed at the age of 5 years, 954 children assessed at age 7,955 children assessed at age 9 , and 925 children assessed at age 11 . McGee (1985) compared children who were lost to the study at each age with those who remained at age 11 and found no significant differences for social class or for a variety of cognitive (including the WISCR) and behavioral variables. When compared with the New Zealand general population, the cohort was slightly biased toward higher socioeconomic status levels. The cohort was also predominantly of European descent and was underrepresentative of children from Polynesian background relative to their incidence in the New Zealand population.

\section{Measures}

The wISC-R was administered at the ages of 7,9 , and 11 years according to standard protocol, except for the omission of the Comprehension and Picture Arrangement subtests because of time constraints on the assessment program. Verbal and performance IQs were prorated using the method recommended in the test manual (Wechsler, 1974). The PIQ was subtracted from the VIQ to obtain the VIQ-PIQ discrepancy score; a positive VIQ-PIQ means VIQ is greater than PIQ, and a negative score indicates the reverse pattern.

Behavior problems were assessed at the ages of 7,9, and 11 years using the Rutter Behavior Problem Checklist Child Scale A for parents and Child Scale B for teachers (Rutter, Tizard, \& Whitmore. 1970). The Burt Word Reading Test (Scottish Council for Research in Education, 1976) was administered at ages 7,9 , and 11 as a word-recognition measure of reading skill. Spelling was evaluated at ages 9 and 1 i hy use of a 25-item spelling test (Silva, Smith, \& Pcarce, 1984). At age 1I, scores on the Progressive Achievement Tests (PAT) for reading comprehension, reading vocabulary, listening comprehension, and mathematics skills (Elley \& Reid, 1969; Elley \& Reid, 1971; Reid \& Hughes, 1974) were available for 616 of the cohort children. These data were collected in a broader study of educational achievement, and no significant differences were found between the members of the sample cohort and children of the same age from the community at large (Silva, 1984). Motor skills were evaluated at ages 7 and 9 using the Basic Motor Ability Tests (BMAT), following the procedures described by Arnheim and Sinclair (1974). At age 11, motor skills were evaluated using a subset of the tasks found to be most discriminatory from previous assessments. These measures of behavior problems, academic achievement, and motor skills were selected for use with the cohort based on high estimates of reliability and validity cited in their respective references.

Perinatal data were recorded from the children's medical records shortly after birth. Silva, McGee, and Williams (1984) have listed the perinatal problems that were recorded. Children who had been characterized by one or more of these problems (e.g., low hirth weight for gestational age, twin status, low Apgar score) were designated as having a positive history of perinatal difficulty $(n=156)$.

Early childhood neurological abnormalities were assessed by combining information from neurological examinations of the children that were conducted at birth and at the ages of 3 and 5 years. At birth, the infants were noted as having minor or major neurological abnor malities as a part of the perinatal medical examination. At 3 years of age, the children received a neurological examination comprising 40 items derived from the procedures described by Touwen and Prechtl (1970). Based on the results of this examination, children were divided into groups with no abnormalities or very mild abnormalities and groups 
with "fairly obvious neurological abnormalities but considered unlikely to have a major effect on functioning" or with "major neurological abnormalities associated with severe impairment of function" (Silva, 1976, p. 19.5). Using the same procedures, we examined 228 children from the sample for neurological abnormalities at the age of 5 years. With the use of $20 \%$ random sampling, we selected 181 children for repeat examination, and the 47 children who showed one or more signs at the age of 3 years were also included. Because gross neurological abnormalities were usually stable, the 5 children so affected at age 3 were not reexamined at age 5 (McGee, Clarkson, Silva, \& Williams, 1982). Any child who showed more than four neurological signs at age 5 (25 of the 228) was designated by the first author as evidencing neurological abnormality. To combine the results from these three evaluations, any child with minor or major abnormalities at birth, with obvious or major abnormalities at age 3 , or with four or more signs at age 5 was considered to have a positive history of early childhood neurological abnormality $(n=67)$.

At each assessment age $(3,5,7,9$, and 11 years) mothers were asked to provide details on the accidental injuries experienced by their children that resulted in medical attention being sought (Langley, Silva, \& Williams, 1981). From these pools of information, all reports of injury to the head were selected based on the following criteria: (a) the word concussion was specifically mentioned or two or more postconcussional symptoms (loss of consciousness; vomiting; blurred vision; clouded consciousness; severe headache; retrograde amnesia; or subsequent fatigue, drowsiness, or depression) were described and (b) the child was seen for emergency treatment at a hospital. The mothers' reports were retrospective over 2-year periods, and therefore they were subject to recall error. The reports from the ages of 9 and 11 years for children from the sample who lived in the catchment area of the Dunedin Public Hospital were checked for validity against hospital records of emergency room treatment and admission, and agreement was excellent. (Cases listed by the hospital that the mothers had failed to report were added to the data.) Frequency of the reported concussion cases showed 10 cases at the age of 3 years, 8 cases at age 5, 12 cases at age 7, 17 cases at age 9 , and 24 cases at age 11 . Several children had experienced concussion at more than one age; the total of those who had experienced concussion by age 11 was 67 . (The number of concussion cases varies across the WISC-R test ages because cases were only added to the analyses at ages subsequent to the date of the mother's report of injury.)

The last measure developed assessed health problems of neurological significance. Repeated medical examinations of the child and parental reports of the child's health were obtained systematically at the biyearly assessments. These sources and medical-record searches yielded documentation of severe health problems of this type for several children. These cases were selected by the first author based on a criterion of a clear record of neurological diagnosis or symptomatology by at least one physician. In all cases, the health problems were reported by more than one source or at more than one assessment age. Because the few cases of discrete syndromes could not support independent analysis, these cases were combined to form a single, dichotomously coded variable. Nineteen children had experienced a relatively severe head injury. For example, one girl who was struck by a motor vehicle at the age of 5 years continued to suffer a residual left hemiplegia, speech disorder, disinhibited social behavior, and grand mal seizures. Congenital syndromes that show neurological symptoms, such as cerebral palsy, Down's syndrome, and cortical blindness, characterized 17 children. Seizure disorders that required medication (febrile seizures were excluded) were recorded in 15 medical histories. Ten children were known to evidence obvious hemiplegias or strongly lateralized neurological findings. Major gross neurological abnormalities that had been assessed in early childhood characterized 14 children. Seven children were under the care of a physician for severe, longstanding migraine headaches. The cumulative number of subjects who showed significant neurological health problems was 51 . Many of these cases met more than one criterion for inclusion, such as the case previously described with severe head injury, hemiplegia, and seizure disorder. In addition, the number for this measure varied across the three wISC-R assessment ages because head injury cases were only added at the ages following the date of injury and because some of the most multiply handicapped children became less accessible for intelligence testing as they grew older. It is acknowledged that health problems that are not directly neurological (such as respiratory disease) may have implications for brain function, but these disorders were not considered because they did not meet the criterion for physician documentation of neurological symptomatology. Medications were not recorded systematically and, thus, were not used in case designation.

Because of the relatively large number of statistical analyses, the Bonferroni inequality (Grove \& Andreason, 1982) was used to control for an inflated Type 1 error rate. For example, the six dependent measures at the age of 11 years that are reported in Table 3 were individually examined, and with $\alpha=.05 \div 6=.0083$. This effectively held the overall Type I error rate for this family of measures to $\alpha=.05$.

\section{Results}

Statistical abnormality was defined to include VIQ-PIQ discrepancy scores beyond the 90th percentile in absolute value. The criterion yielded cut-off scores at the age of 7 years of 22 IQ points ( 94 children), at age 9 of 23 IQ points ( 88 children), and at age 11 of 24 IQ points ( 89 children). These scores at the 90th percentile rank are almost identical to those provided by Kaufman (1980) (full standardization sample, 22 IQ points; children of professional and technical workers, 23 IQ points). Two groups, VIQ-PIQ and comparison, were defined at each of the three test ages as either above or below the 90 th percentile VIQ-PIQ scores.

The absolute value of the VIQ-PIQ score discrepancy (regardless of discrepancy direction) was not significantly correlated with WISC-R Full Scale IQ score at the age of 7 years $(r=.028$, $p>.05)$, at age $9(r=.048, p>.05)$, or at age $11(r=.042$, $p>.05)$. Thus, VIQ-PIQ discrepancies were considered without reference to level of performance.

\section{Clinical Significance of VIQ-PIQ Score Discrepancies}

Possible etiological factors. It was hypothesized that VIQPIQ discrepant children would be characterized by a greater incidence of factors suggesting possible brain insult or disorder than would control children at each age. Table 1 provides comparison of the groups on four factors that are possibly associated with some level of brain dysfunction: perinatal problems, early childhood neurological abnormality, health problems of neurological significance, and concussion. The categorical nature of these possible etiological variables required the use of chisquare tests for significance of differences in group rates. The VIQ-PIQ score discrepancy was not associated with significantly elevated rates of any factor at any of the three ages.

Possible sequelae. It was hypothesized that VIQ-PIQ children would be characterized by a greater frequency, relative to control children, of problems in academic achievement, behavior, or motor skills. These three variables were assessed in scale format, which required the application of Student's $t$ tests to the differences between group mean scores. Table 2 presents group mean scores and standard deviations for the possible se- 
Table 1

Group Percentages of Four Possible Central Nervous System Insult Indices for Children With and Without WISC-R VIQ-PIQ Discrepancy at Ages 7.9, and 11

\begin{tabular}{|c|c|c|}
\hline Variable & VIQ-PIQ $^{a}$ & Comparison $^{b}$ \\
\hline \multicolumn{3}{|c|}{ Age 7} \\
\hline Perinatal problems & 2.3 & 1.4 \\
\hline $\begin{array}{l}\text { Early childhood neurological } \\
\text { abnormalities }\end{array}$ & 0.7 & 0.6 \\
\hline $\begin{array}{l}\text { Health problems of neurological } \\
\text { significance }\end{array}$ & 7.4 & 6.1 \\
\hline Concussion & 1.1 & 3.3 \\
\hline \multicolumn{3}{|c|}{ Age 9} \\
\hline Perinatal problems & 1.7 & 1.5 \\
\hline $\begin{array}{l}\text { Early childhood neurological } \\
\text { abnormalities }\end{array}$ & 0.7 & 0.6 \\
\hline $\begin{array}{l}\text { Health problems of neurological } \\
\text { significance }\end{array}$ & 1.1 & 7.2 \\
\hline Concussion & 8.0 & 4.0 \\
\hline \multicolumn{3}{|c|}{ Age 11} \\
\hline Perinatal problems & 2.1 & 1.5 \\
\hline $\begin{array}{l}\text { Early childhood neurological } \\
\text { abnormalities } \\
\text { Health problems of neurological }\end{array}$ & 0.5 & 0.6 \\
\hline significance & 5.6 & 7.2 \\
\hline Concussion & 5.6 & 7.0 \\
\hline
\end{tabular}

Note. WISC-R = Wechsler Intelligence Scale for Children-Revised; VIQ = verbal IQ; PIQ = performance IQ. The viQ-PIQ group was defined by scores beyond the 90th percentile at each age. Group sample sizes varied with each analysis because of missing data. Chi-square results were nonsignificant at $p=.05$.

Approximate sample sizes were 94 at age 7,88 at age 9, and 89 at age 11.

'Approximate sample sizes were 897 at age 7,867 at age 9 , and 836 at age II.

quelae of large VIQ-PIQ score discrepancy. No significant group differences were found for mean number of behavior problems, as rated by teachers or parents, at any of the three ages. At the age of 7 years, Burt reading scores, the only measure of academic achievement, were not significantly different for the two groups. Similarly, no significant group differences emerged at age 9 for reading or spelling. At age 11, the VIQ-PIQ children had significantly lower scores than control subjects on two measures of oral reading vocabulary. The groups were also compared on motor skills tasks administered at ages 7, 9, and 11, but no significant group differences were found for any age.

In summary, several possible factors were considered that might have served as etiological bases for the VIQ-PIQ score discrepancy, but none were found to differentiate the VIQ-PIQ group from the control group. Perinatal problems, early neurological abnormalities, concussion, and other health problems of likely neurological significance did not characterize the VIQPIQ subjects in elevated rates. Possible adverse sequelae for VIQPIQ subjects were also examined. No group differences at any age were found in behavior or motor skills problems, but problems with reading did differentiate the groups at age 11 .

\section{Direction of the VIQ-PIQ Discrepancy}

Cases with VIQ-PIQ scores above the 90th percentile were not found to be evenly distributed in direction of the discrepancy. At the age of 7 years, $61 \%$ of the 94 VIQ-PIQ children had PIQ scores greater than VIQ scores. At age $9,62 \%$ of the 88 VIQ-PIQ subjects showed this pattern with depressed VIQ scores. At age $11,85 \%$ of the 89 VIQ-PIQ group members had PIQ scores higher than their VIQ scores.

Within the VIQ-PIQ cases at each age, VIQ greater than PIQ and PIQ greater than VIQ groups were compared on the same indicators of clinical significance reported previously in order to investigate whether one directional pattern or the other was more likely to be associated with any of the possible etiological or adverse sequelae factors. At no age were VIQ greater than PIQ scores or PIQ greater than VIQ scores found to be differentially associated with rate of concussion, health problems of neurological significance, early childhood neurological abnormalities, or perinatal problems. All chi-square values were non-

Table 2

Mean Group Scores for Behavioral, Academic, and Motor Problems for Children With and Without VIQ-PIQ Discrepancy at Ages 7, 9, and 11

\begin{tabular}{|c|c|c|c|c|}
\hline \multirow[b]{2}{*}{ Variable } & \multicolumn{2}{|c|}{ VIQ-PIQ } & \multicolumn{2}{|c|}{ Comparison } \\
\hline & $M$ & $S D$ & $M$ & $S D$ \\
\hline \multicolumn{5}{|c|}{ Age 7} \\
\hline $\begin{array}{l}\text { Teachers' report of behavior } \\
\text { problems }\end{array}$ & 2.0 & 2.0 & 1.8 & 1.8 \\
\hline Parents' report of behavior & & & & 5 \\
\hline $\begin{array}{l}\text { problems } \\
\text { Burt Word Reading }\end{array}$ & $\begin{array}{r}9.8 \\
29.5\end{array}$ & $\begin{array}{r}6.3 \\
16.2\end{array}$ & $\begin{array}{r}8.8 \\
29.5\end{array}$ & $\begin{array}{r}5.5 \\
12.9\end{array}$ \\
\hline BMAT Motor $Z$ score & -0.1 & 0.7 & 0.0 & 0.5 \\
\hline \multicolumn{5}{|c|}{ Age 9} \\
\hline $\begin{array}{l}\text { Teachers' report of behavior } \\
\text { problems }\end{array}$ & 7.5 & 5.5 & 8.1 & 5.4 \\
\hline $\begin{array}{l}\text { Parents' report of behavior } \\
\text { problems }\end{array}$ & & & & 51 \\
\hline Burt Word Reading & $\begin{array}{r}3.8 \\
54.1\end{array}$ & $\begin{array}{r}4.1 \\
19.6\end{array}$ & $\begin{array}{r}4.3 \\
53.8\end{array}$ & $\begin{array}{r}3.1 \\
19.0\end{array}$ \\
\hline Spelling & 9.9 & 6.0 & 10.4 & 6.1 \\
\hline BMAT Motor $Z$ score & 0.1 & 0.5 & 0.0 & 0.5 \\
\hline
\end{tabular}

Age 11

Teachers' report of behavior problems

Parents' report of behavior problems

Burt Word Reading

Spelling

PAT Reading Comprehension

PAT Reading Vocabulary

PAT Listening Comprehension

PAT Mathematics

BMAT Motor $Z$ score

$\begin{array}{rcrr}4.1 & 4.9 & 3.8 & 5.0 \\ 6.8 & 4.9 & 7.1 & 5.4 \\ 66.6 & 18.2^{*} & 73.1 & 20.3 \\ 14.3 & 5.5 & 15.9 & 5.7 \\ 44.4 & 28.0 & 51.9 & 28.1 \\ 40.8 & 28.0^{*} & 51.6 & 27.4 \\ 44.2 & 29.4 & 52.3 & 27.4 \\ 43.0 & 27.0 & 46.8 & 26.5 \\ 0.0 & 0.6 & 0.0 & 0.6\end{array}$

Note VIQ = verbal IQ; $\mathrm{PIQ}=$ performance IQ. BMAT $=$ Basic Motor Ability Tests; $P A T=$ Progressive Achievement Tests. The VIQ-PIQ group was defined by scores beyond the 90 th percentile at each age.

${ }^{*} p<.05$ according to $t$-test results. 
Table 3

Mean Academic Achievement Scores of Groups With VIQ Greater Than PIQ and PIQ Greater Than VIQ

Patterns at Ages 7, 9, and 11

\begin{tabular}{|c|c|c|c|c|}
\hline \multirow[b]{2}{*}{ Variable } & \multicolumn{2}{|c|}{$\mathrm{VIQ}>\mathrm{PIQ}$} & \multicolumn{2}{|c|}{$\mathrm{PIQ}>\mathrm{VIQ}$} \\
\hline & $M$ & $S D$ & $M$ & $S D$ \\
\hline \multicolumn{5}{|l|}{ Age ? } \\
\hline Burt Word Reading & 38.4 & $16.7^{*}$ & 23.9 & 13.3 \\
\hline \multicolumn{5}{|l|}{ Age 9} \\
\hline Burt Word Reading & 66.9 & $20.4^{*}$ & 46.4 & 14.6 \\
\hline Spelling & 13.4 & $5.9^{*}$ & 7.8 & 5.2 \\
\hline \multicolumn{5}{|l|}{ Age 11} \\
\hline Burt Word Reading & 80.4 & $17.0^{*}$ & 64.3 & 17.4 \\
\hline Spelling & 17.0 & 5.6 & 13.9 & 5.4 \\
\hline PAT Reading Comprehension & 82.3 & $21.5^{*}$ & 38.6 & 24.0 \\
\hline PAT Reading Vocabulary & 75.4 & $28.0^{*}$ & 35.6 & 24.2 \\
\hline PAT Listening Comprehension & 80.6 & $23.8^{*}$ & 38.2 & 25.9 \\
\hline PAT Mathematics & 71.6 & $27.9^{*}$ & 38.6 & 24.1 \\
\hline
\end{tabular}

Note. VIQ $=$ verbal IQ; PIQ $=$ performance IQ. PAT $=$ Progressive Achievement Tests. The VIQ-PIQ group was defined by scores beyond the 90th percentile at each age.

${ }^{*} p<.05$ according to $t$-test results.

significant. Similarly, at none of the three ages was the direction of VIQ-PIQ discrepancy differentially associated with teacher or parent reports of behavior problems or with motor skills. In contrast, the PIQ greater than VIQ pattern was markedly associated with poor academic achievement at all three ages. Academic-achievement-scale mean scores for the two directional VIQ-PIQ groups are presented in Table 3. Tests of reading, spelling, and mathematics all yielded mean scores for the PIQ greater than VIQ group at a standard deviation below the mean for the VIQ greater than PIQ group.

In summary, at each of three test ages, depressed VIQ scores relative to PIQ scores was the most frequent pattern among children whose VIQ-PIQ discrepancy scores were beyond the 90th percentile in extremity. This pattern, although not notably associated with any of the possible brain disorder factors available for this study, was strongly associated with deficits in academic achievement.

\section{Stability of VIQ-PIQ Discrepancy Across Ages}

Repeated WISC-R testing at 2-year intervals presented an opportunity to examine longitudinally the stability of the VIQ-PIQ discrepancy. The VIQ-PIQ discrepancy scores between test ages were positively correlated, with temporally adjacent test ages having slightly higher correlations. At the ages of 7 and 9 years, the VIQ-PIQ yielded Pearson $r=.52(p<.01)$; at ages 9 and 11 , the VIQ-PIQ scores yielded Pearson $r=.62(p<.01)$; and at ages 7 and 11 , the VIQ-PIQ yielded $r=.50(p<.01)$. Despite these reasonably high correlations, the majority of children whose VIQ-PIQ scores were beyond the 90th percentile at one age did not maintain such a large discrepancy at the next age (or had not evidenced extreme VIQ-PIQ discrepancy at the previous age), a finding in close agreement with Bishop and Butterworth (1980). Of the VIQ-PIQ subjects at age 7,21\% remained in the VIQ-PIQ group at age 9 . Twenty-five percent of the original 88 children still maintained VIQ-PIQ scores of between 16 and 23 IQ points at age 9 and, therefore, maintained a notable amount of VIQ-PIQ discrepancy, although this amount was not sufficient to meet the 90th percentile criterion for 9-year-olds. This rate of relative stability, nevertheless, reveals that more than half of the extreme VIQ-PIQ 7-year-olds had VIQ-PIQ scores well within normal limits by age 9 . The percentages of children who remained stable and children who normalized VIQ-PIQ status between ages 9 and 11 were almost identical to those described between ages 7 and 9 .

It is possible that the children whose extreme VIQ-PIQ scores remained relatively stable over the 2-year time periods in this study were distinct in some ways from children with unstable discrepancy scores. A criterion of having been in the VIQ-PIQ group at more than one age ( 7 and 9,9 and 11,7 and 11 , or all three ages) yielded 49 cases. The ratio of VIQ greater than PIQ to PIQ greater than VIQ among these stable cases was similar to that reported earlier for the individual test ages; about $75 \%$ of the 49 stable VIQ-PIQ cases showed the PIQ greater than VIQ pattern. Eighteen children evidenced VIQ-PIQ scores beyond the 90th percentile across all three ages, and 17 of these were cases with the PIQ greater than VIQ pattern. Therefore, although neither pattern seemed more likely to remain stable across age levels, the depressed VIQ pattern did appear to remain stable across more ages, that is, for a longer period of time.

The 49 stable cases were compared with the remaining VIQPIQ cases (nonstable VIQ-PIQ cases with WISC-R data from three test ages were combined, $n=168$ ) to examine the hypothesis that stable VIQ-PIQ cases can be distinguished by their etiological histories or by some adverse sequelae. Stable VIQ-PIQ cases were not significantly different from nonstable cases in rates of perinatal problems, early neurological abnormalities, health problems of neurological significance, or concussion. Neither were the two groups different in mean motor skills scores obtained at any age.

Both parents and teachers had reported an average of more behavior problems for the stable VIQ-PIQ children than for the nonstable group at all three ages, but no differences were significant. Mean academic achievement scores for the stable group were lower than those for the nonstable group at all three ages as well. These differences were significant only for the Burt Reading Test at age $11, t(206)=3.27, p<.01$, and for the PAT test of reading vocabulary at age $11, t(139)=2.81, p<.01$.

On the whole, VIQ-PIQ discrepancy did not remain a stable characteristic across childhood. Cases in which VIQ-PIQ discrepancy was maintained for 2 or more years had a preponderance of the PIQ greater than VIQ pattern, as did nonstable cases, and this pattern appeared to remain stable for more years than the reverse pattern. Stable cases did not seem to be distinguished by possible etiological-disorder factors, but they were distinguished by a marginally greater number of academic problems.

\section{Discussion}

This investigation tested the hypothesis that a statistically abnormal VIQ-PIQ score signals clinically abnormal status in neurological history, behavior, or achievement for a child from the 
general population. Several variables, both prospective and concurrent, were examined that might have served as etiological contributors to cognitive dysfunctions that produce a wide VIQ-PIQ discrepancy. Neither perinatal difficulties, early childhood neurological abnormalities, health problems of neurological significance, nor concussion proved to be more prevalent among children with VIQ-PIQ discrepancy than among children without such discrepancy. Prospective collection of the data for these etiological variables had the advantage of reducing error and bias. Nevertheless, a disadvantage was that the data were not deliberately collected for the purpose of this investigation. More-detailed medical histories may have allowed finer distinctions in determining caseness, especially regarding histories of concussion and of health problems such as congenital disorders or epilepsy. More-inclusive criteria for caseness may have yielded different results. On the other hand, less-detailed and less-reliable historical information than that used in this study often comes to the desk of a psychologist requested to assess a child.

The VIQ-PIQ discrepant cases were also examined for elevated rates of difficulties that may develop from the types of cognitive deficits that yield a VIQ-PIQ discrepancy. Neither behavior problems nor motor problems were found to signifcantly relate to VIQ-PIQ scores. The primary finding is that a pattern of PIQ greater than VIQ scores was notably associated with relatively poor academic achievement at the ages of 7,9, and 11 years. Because PIQ greater than VIQ scores was also a more frequent and a longer lasting pattern than VIQ greater than PIQ scores, the potential for its use as a flag for academic difficulties has gained interest. Satz (1976) and Bryant and Bradley (1985) have provided excellent discussions of the association of depressed VIQ with learning disability.

The relatively greater frequency of the PIQ greater than VIQ pattern at all three ages was an unexpected finding. It is, of course, possible that this prevalence pattern is unique to New Zealand children. However, several verbal wISC-R items were altered for local relevance (e.g., "Who discovered New Zealand?'), making it unlikely that the prevalence of depressed verbal scores is the result of inordinate item difficulty. In fact, New Zealand children scored an average of 5 WISC-R IQ points higher than American children, and this score elevation was found for the Verbal and Performance subtests alike (Silva, 1982).

Results from Russell (1979) and Dennis (1985) on the IQ effects of distinct brain damage loci and disease processes have suggested that, in general, VIQ is depressed by left hemisphere damage, whereas PIQ is depressed by both right hemisphere and central or diffuse damage. The specificity of this VIQ effect suggests that depressed VIQ should be the less frequent discrepancy pattern. Indeed, Kolb and Whishaw (1985) stated that "it is rare to obtain a relatively low verbal IQ, and its appearance should not be ignored" (p. 674). Relatively low VIQ was not the rare pattern in this sample. Thus, the PIQ greater than VIQ pattern may be a useful lateralizing sign among well-documented clinical cases, but it does not seem to be a good discriminator for less clear-cut cases from the general population.

The VIQ-PIQ score discrepancy did not remain a stable characteristic across childhood for the majority of children studied. The absence of such stability was predicted from the clinical literature, wherein the VIQ-PIQ score discrepancy is described as an acute effect of brain insult (Bishop \& Butterworth, 1980; Levin, Benton, \& Grossman, 1982; Pihl, 1968; Russell, 1979). Nevertheless, in this sample, a noticeable number of children (about 5\% of the total cohort) did maintain wide VIQ-PIQ discrepancies for at least 2 years or more, and this pattern was maintained in the absence of any documented cerebral insult or disease process. Therefore, although a wide VIQ-PIQ discrepancy has been shown to be acutely associated with brain insult in studies of clinical cases (e.g., Dennis, 1985), a wide VIQ-PIQ discrepancy cannot be used clinically as a presumptive indicator of brain disorder of recent onset.

The results of this investigation support Rutter's (1983) assertion that VIQ-PIQ discrepancies are of doubtful diagnostic value given the differential base rates of brain disorder and VIQPIQ discrepancy in the population. Previous studies of clinical cases have shown VIQ-PIQ discrepancy to be acutely associated with brain insult and PIQ greater than VIQ scores to be the rarer of the two possible discrepancy patterns. In contrast, the present study of an unselected cohort found about $23 \%$ of VIQPIQ cases to retain the VIQ-PIQ discrepancy for at least 2 years and found PIQ greater than VIQ scores to be the most common pattern. These contrasts suggest that the VIQ-PIQ discrepancy in the general population is not the same sign that it is in patients with documented brain disorder. It is probably not advisable to interpret the VIQ-PIQ discrepancy in a general, judicial, or educational setting as one might interpret it in a neurological setting. As a general rule, the VIQ-PIQ discrepancy might best be interpreted as an adjunct to "other measures of mental functions that have been examined within the context of the patient's life experiences, current psychosocial situation, and the medical history" (Lezak, 1983, p. 253).

\section{References}

Arnheim, D., \& Sinclair, W. (1974). The clumsy child. St. Louis, MO Mosby.

Bishop, D. V. M., \& Butterworth, G. E. (1980). Verbal-performance discrepancies: Relationship to birth risk and specific reading retardation. Cortex, 16, 375-389.

Black, F. W. (1974). WISC Verbal-performance discrepancies as indicators of neurological dysfunction in pediatric patients. Journal of Clinical Psychology, 30, 165-167.

Bortner, M., Hertzig, M. E., \& Birch, H. G. (1972). Neurological signs and intelligence in brain-damaged children. Journal of Special Education, 6, 325-333.

Braun, J. S., \& Brane, M. (1971). Comparison of performance of children with dysrhythmia Grade 1 and normal EEG on psychological tests. Proceedings of the 79th Annual Convention of the American Psychological Association, 6, 457-458.

Bryant, P., \& Bradley, L. (1985). Children's reading problems. Oxford. England. Blackwell.

Caputo, D. V., Edmonton, W. E., L'Abate, L., \& Rondberg, S. R. (1963) Type of brain damage and intellectual functioning in children. Journal of Consulting Psychology, 27, 1984.

Cronbach, L. J. (1961). Essentials of psychological testing New York: Harper \& Row.

Dennis, M. (1985). Intelligence after early brain injury: II. IQ scores of subjects classified on the basis of medical history variables. Journal of Clinical and Experimental Neuropsychology, 7, 555-576.

Dennis, M., Fitz, C. R., Netley, C. T., Sugar, J., Harwood-Nash. 
D. C. F., Hendrick, E. B., Hoffman, H. J., \& Humphreys, R. P. (1981). The intelligence of hydrocephalic children. Archives of Neurology, 38, $607-615$.

Elley, W. B., \& Reid, N. A. (1969). Progressive Achievment Tests teacher's manual: Reading comprehension and reading vocabulary. Wellington, New Zealand: Council for Educational Research.

Elley, W. B., \& Reid, N. A. (1971). Progressive Achievement Tests teacher's manual: Listening comprehension. Wellington, New Zealand: Council for Educational Research.

Fedio, P., \& Mirsky, A. F. (1969). Selective intellectual deficits in children with temporal lobe or centrecephalic epilepsy. Neuropsycholgia, 7, 287-300.

Grove, W. M., \& Andreason, N. C. (1982). Simultaneous tests of many hypotheses in exploratory research. Journal of Nervous and Mental Disorder, 170, 3-8.

Guertin, W. H., Ladd, C. E., Frank, G. H., Rabin, A. I., \& Hiester, D. S. (1966). Research with the Wechsler Intelligence Scale for Adults: 1960-1965. Psychological Bulletin, 66, 385-409.

Holroyd, J. (1968). When WISC Verbal IQ is low. Journal of Clinical Psychology, 24, 457.

Kane, R. L., Parsons, O. A., \& Goldstein, G. (1985). Statistical relationships and discriminative accuracy of the Halstead Reitan, Luria Nebraska, and Wechsler IQ scores in the identification of brain damage. Journal of Clinical and Experimental Neuropsychology, 7, 211-223.

Kaufman, A. S. (1980). Issues in psychological assessment: Interpreting the wISC-R intelligently. In B. B. Lahey \& A. E. Kazdin (Eds.), $A d-$ vances in clinical child psychology (Vol. 3, pp. 177-214). New York: Plenum Press.

Klove, H. (1959). Relationship of differential electroencephalographic patterns to distribution of Wechsler-Bellevue scores. Neurology, 9 , 871-876.

Klove, H., \& Reitan, R. M. (1958). Effect of dysphasia and distortion on Wechsler-Bellevue results. Archives of Neurology and Psychiatry, $80,708-713$.

Kolb, B., \& Whishaw, I. Q. (1985). Fundamentals of human neuropsychology (2nd ed.). New York: W. H. Freeman.

Langley, J. D., Silva, P. A., \& Williams, S. (1981). Accidental injuries in the sixth and seventh years of tife: A report from the Dunedin Multidisciplinary Child Development Study. New Zealand Medical Journal, 93, 344-347.

Levin, H. S., Benton, A. L., \& Grossman, R. G. (1982). Neurobehavioral consequences of closed head injury. New York: Oxford University Press.

Lezak, M. D. (1983). Neuropsychological assessment. New York: Oxford University Press.

Matarazzo, J. D. (1972). Wechsler's measurement and appraisal of adult intelligence (5th ed.). Baltimore, MD: Williams \& Wilkins.

McGee, R. (1985). Response rates of Phase XI of the Dunedin Multidisciplinary Health and Development Study. Unpublished report, Dunedin Multidisciplinary Health and Development Research Unit, Otago Medical School, Dunedin, New Zealand.

McGee, R., Clarkson, J. E., Silva, P. A., \& Williams, S. (1982). Neurological dysfunction in a large sample of three-year-old children: A report from the Dunedin Multidisciplinary Child Development Study. New Zealand Medical Journal, 95, 693-696.

McGee, R., \& Silva, P. A. (1982). A thousand New Zealand children: Their health and development from birth to seven (Special Rep. Series No. 8). Auckland: Medical Research Council of New Zealand.

Pihl, R. O. (1968). The degree of verbal-performance discrepancy on the WISC and the WAIS and severity of abnormality in epileptics. Journal of Clinical Psychology, 24, 418-420.

Reid, N. A., \& Hughes, D. C. (1974). Progressive Achievement Tests teacher's manual: Mathematics. Wellington, New Zealand: Council for Educational Research.

Reitan, R. M. (1955). Certain differential effects of left and right cerebral lesions in human adults. Journal of Comparative and Physiological Psychology, 48, 474-477.

Russell, E. W. (1979). Three patterns of brain damage on the WAIS. Journal of Clinical Psychology, 35, 611-620.

Rutter, M. (Ed.). (1983). Developmental neuropsychiatry. New York: Guilford Press.

Rutter, M., Graham, P., \& Yule, W. (1970). A neuropsychiatric study in childhood (clinics in developmental medicine nos. 35-36). London: Spastics International Medical Publications/Heinemann Medical Books.

Rutter, M., Tizard, J., \& Whitmore, K. (1970). Education, health and behavior. London: Longmans.

Satz, P. (1976). Cerebral dominance and reading disability: An old problem revisited. In R. M. Knights \& D. J. Bakker (Eds.), The neuropsychology of learning disorders (pp. 273-297). Baltimore, MD: University Park Press.

Scottish Council for Research in Education. (1976). The Burt Word Reading Test: 1974 revision. London: Hodder \& Stoughton.

Silva, P. A. (1976). A thousand Dunedin three-year-olds: A multidisciplinary study of child development. Unpublished report, Medical Research Council of New Zealand.

Silva, P. A. (1982). Interpreting Stanford-Binet and WISC-R IQs in New Zealand: Research notice. New Zealand Journal of Educational Studies, $17,195$.

Silva, P. A. (1984). The educational attainment of 2,600 Dunedin children: $A$ report on the Dunedin intermediate schools' Collaborative Testing Project 1984: The NZCER tests (Monograph). Dunedin, New Zealand: Otago Medical School.

Silva, P. A., McGee, R., \& Williams, S. (1984). A seven-year followup study of the cognitive development of children who experienced common perinatal problems. Australian Paediatric Journal, 20. 2328.

Silva, P. A., Smith, C. T. W., \& Pearce, D. W. (1984). The Dunedin Spelling Tests. Dunedin, New Zealand: Dunedin Multidisciplinary Health and Development Research Unit, Department of Paediatrics and Child Health, University of Otago Medical School.

Todd, J., Coolidge, F., \& Satz, P. (1977). The Wechsler Adult Intelligence Scale discrepancy index: A neuropsychological evaluation. Journal of Consulting and Clinical Psychology, 45, 450-454.

Touwen, B. C., \& Prechtl, H. R. (1970). The neurological examination of the child with minor nervous dysfunction. London: Heinemann Medical Books.

Uzzell, B. P., Zimmerman, R. A., Dolinskas, C. A., \& Obrist, W. D. (1979). Lateralized psychological impairment associated with $C T$ lesions in head injured patients. Cortex, 15, 391-401.

Wallace, S. J., \& Cull, A. M. (1979). Long-term psychological outlook for children whose first fit occurs with fever. Developmental Medicine and Child Neurology, 21, 28-40.

Wechsler, D. (1974). Manual of the Wechsler Intelligence Scale for Children-Revised. New York: Psychological Corporation.

Wechsler, D. (1981). Manual of the Wechsler Adult Intelligence ScaleRevised. New York: Psychological Corporation.

Received August 25, 1986

Revision received January 29, 1987

Accepted February 9,1987 